\title{
Cloud phase identification based on brightness temperatures provided by the bi-spectral IR Camera of JEM-EUSO Mission
}

\author{
Antonio J. de Castro, Susana Briz ${ }^{a}$, Isabel Fernández-Gómez, Irene Rodríguez, \\ and Fernando López for JEM-EUSO Collaboration \\ Universidad Carlos III de Madrid, Physics Department, Avda. de la Universidad 30, 28911 Leganés (Madrid), Spain
}

\begin{abstract}
Cloud information is extremely important to correctly interpret the JEM-EUSO telescope data since UV radiation coming from the Extensive Air Shower can be partially absorbed or reflected by clouds. In order to observe the atmosphere and clouds in the field of view of the UV telescope the JEM-EUSO system will include an Atmospheric Monitoring System, which consists of a LIDAR and an IR Camera. Until now several radiative algorithms have been developed to retrieve the cloud top temperature from the brightness temperatures (BT) that the IR Camera will provide in two IR spectral bands $(10.8$ and $12 \mu \mathrm{m})$. In some cases the performance of the algorithms depends on cloud phase: water, ice or mixed. For this reason the identification of the cloud phase is valuable information for the correct interpretation of the cloud temperatures retrieved by radiative algorithms. Some previous proposals based on brightness temperature differences (BTD) have revealed that it is not easy to determine unambiguously the phase. In this work we present criteria to retrieve the cloud phase based on IR Camera BTDs. It has been checked with MODIS images to evaluate the possibilities to identify cloud phase with the JEM-EUSO IR Camera.
\end{abstract}

\section{Introduction}

The knowledge of cloud parameters such as the phase (water, ice or mixed), the emissivity and other cloud propertiesare essential to understand issues as important as the radiative balance which rules drives the global climate change [1-3]. Cloud classifications are also useful to derive information from satellites and for operational meteorology or weather forecasting $[4,5]$. In this work the objective of the cloud classification is to provide useful information for the analysis of the JEM-EUSO IR Camera data, especially for the cloud height retrieval.

The ultimate aim of JEM-EUSO Mission is to identify the sources of Ultra High Energy Cosmic Rays (UHECR) [6]. The main instrument of JEM-EUSO is an UV telescope which will be located on the International Space Station (ISS). However JEM-EUSO telescope will not observe UHECRs directly but instead it will detect the UV radiation produced by the interaction between the UHECRs and the atmosphere (Extensive Air Shower, EAS). The analysis of the UV images, EAS reconstruction, will give information of the UHECR properties (energy, arrival direction, etc.).

However the UV radiation is interfered by the atmosphere and the clouds in the path between the EAS and the telescope which can lead to misreconstruction of the EAS event [7]. On the one hand, high thin clouds (generally ice clouds) can absorb part of the signal coming from the EAS which implies an underestimation of the primary energy UHCER calculated in the reconstruction [7]. On the other hand, Cherenkov light, which is reflected by the Earth surface in clear sky conditions, would be reflected by thick clouds (ice or water clouds) and this fact may lead to an error in the estimation of the depth

a e-mail: sbriz@fis.uc3m.es of maximum development of the EAS [7]. Moreover the effects of those clouds depend on their height.Therefore it is extremely important to know the atmospheric conditions and detect if there are clouds in the Field of View (FoV) of the telescope. Moreover, the cloud top height and the cloud emissivityare essential for an appropriate reconstruction of the EAS.

In order to monitor the required information in the main telescope FoV, the JEM-EUSO mission will implement an Atmospheric Monitoring System (AMS) which will be attached to the main system. The AMS consists of a LIDAR, an infrared (IR) camera and global atmospheric models [8]. The LIDAR will measure the optical depth profiles of the atmosphere in selected directions [9]. The IR camera is expected to provide data suitable to retrieve mainly the cloud coverage and the cloud height, although it could also give some information of the emissivity which is closely related to the optical depth profiles. The global atmospheric models will be used to retrieve the atmospheric parameters (temperature, pressure and humidity vertical profiles) in the monitored region.

The JEM-EUSO IR camera preliminary design involves a bi-spectral system. Consequently, after calibration process, the IR camera will provide the brightness temperature (BT) in two $1-\mu \mathrm{m}$ width spectral bands centred at 10.8 and $12 \mu \mathrm{m}$, hereinafter referred as $\mathrm{BT}_{11}$ and $\mathrm{BT}_{12}$ [10]. From these BTs, Cloud Top Height (CTH) retrieval can be performed using radiometric information or stereovision technique $[11,12]$, although this paper focuses on the first technique. The radiance emitted by the cloud depends mainly on its temperature and emissivity which can be retrieved from the radiance measured by the IR camera. However some hypotheses considered in the retrieval methodology and the accuracy of the results depend on the cloud type. Therefore if the cloud type (phase and emissivity) was known, this information would 
be useful for the analysis and interpretation of the retrieved cloud temperature and emissivity. The objective of the work here presented is to evaluate the capabilities of the IR camera to provide information about the type of cloud from the brightness temperature (BT) measured in its two spectral bands.

Section 2 summarizes the methodology to retrieve the cloud temperature and emissivity and explain the usefulness of knowing the type of cloud to evaluate the retrievals. The brightness temperature difference (BTD) between the two spectral bands of the IR camera has been studied for different types of clouds. The results are evaluated in Sect. 3. A methodology based on BTD and BT limits is proposed in Sect. 4. Section 5 summarizes the main conclusions of this work.

\section{Clod top height retrieval}

The radiation emitted by the cloud is basically related to its emissivity and temperature according to Planck's law. The radiance emitted by the cloud is measured by the sensor (IR camera) and the system retrieves the temperature of the cloud from radiance measurement by a proper inversion of Planck's law. Therefore the radiation given by the IR camera can be used to get the cloud top temperature (CTT).

Afterwards the cloud top height $(\mathrm{CTH})$ can be estimated by using the relationship between the temperature and the height given by the atmospheric vertical profiles. Atmospheric soundings available all over the world and global atmospheric models provide temperature vertical profiles and therefore both can be used to obtain the CTH from the cloud.

However, due to atmospheric effects (absorption/ emission by gases), the IR radiance emitted by the cloud is not the one received by the IR camera. Therefore the temperature retrieved from measured radiance, i.e., the BT is not the temperature of the cloud. Moreover, if the cloud emissivity is lower than 1 , the radiance emitted by the cloud is not exactly the corresponding radiance emitted by the blackbody at the same temperature and then it is necessary to know the emissivity in advance to retrieve the CTT from the BT. Hence it is evident that some algorithms are necessary to correct the atmospheric effects, to calculate the emissivity and then to obtain the cloud temperature from BT. It is also clear that thick clouds are easier to study than thin clouds where the radiative scenario becomes more difficult since many emitters (Earth surface, atmosphere below the cloud, etc.) contribute to the total radiance received by the IR camera. Attending these facts the strategy designed to retrieve the $\mathrm{CTH}$ is depicted in the diagram of Fig. 1.

First of all, the primary data provided by the IR camera, the BT images in both bands, are used as inputs in a procedure based on Look-Up Tables (LUT) to estimate the emissivity of the cloud [13]. Depending on the emissivity value the cloud temperature can be retrieved from two different approaches:

a) If the emissivity of a pixel or an area is the maximum $(\varepsilon=1)$, the RadiativeTransfer Equation (RTE) simplifies notably and the cloud temperature can be retrieved with a Split Window Algorithm

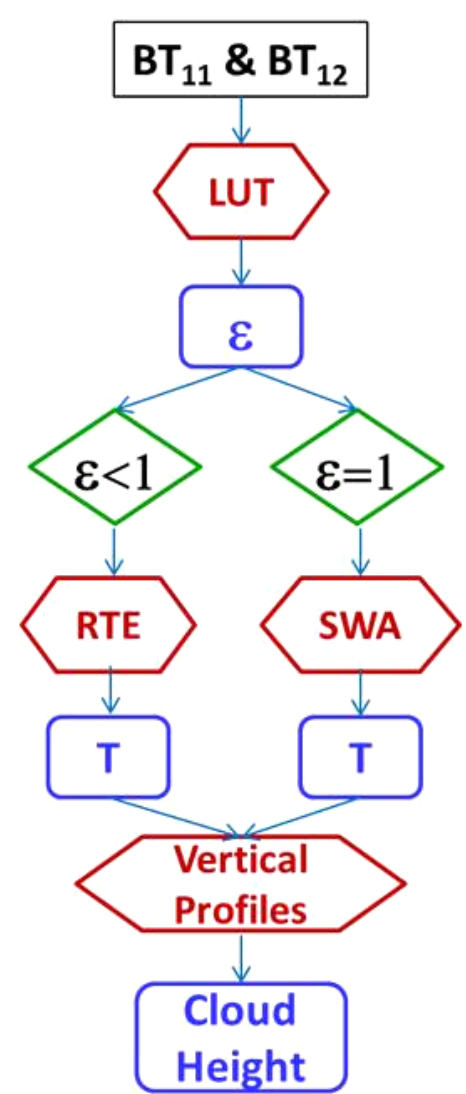

Figure 1. Scheme of the procedure to retrieve the cloud height from the BTs of the JEM-EUSO IR camera. The LUT-based procedure retrieves the emissivity. If the emissivity is 1 a SWA retrieves the cloud temperature. Otherwise other procedure based on an approximation of the RTE is being developed. From the temperature the cloud height can be calculated from temperature vertical profiles.

(SWA) directly from the BTs in two spectral bands [14]. The SWA does not require ancillary information and therefore it is very fast and its accuracy very good.The algorithm was calculated with an approximation of the RTE, considering the maximum emissivity $(\varepsilon=1)$, and simulations of atmospheric radiance with different vertical atmospheric profiles of temperature, humidity, etc. The SWA was validated with simulated scenarios in different atmospheric conditions. The error was higher for low clouds and humid atmospheres but even so it was lower than $0.3 \mathrm{~K}$. The SWA was also applied to real scenarios (MODIS images in spectral bands 31 and 32). The difference between the temperature retrieved by the SWA and this one provided by MODIS waslower than $1 \mathrm{~K}$ for thick water clouds $(\varepsilon=1)$ [14].

b) If the emissivity of a pixel or an area is lower than 1, then the RTE does notsimplify directly, although some approximations can be assumed. At this moment the validity of these approximations is under study. However the LUT-based procedure to retrieve emissivities ofthin clouds $(\varepsilon<1)$ has been validated in simulations and applied to real scenarios (MODIS images). In general it can be stated that the errors in simulations are lower than 0.1 [13]. When 


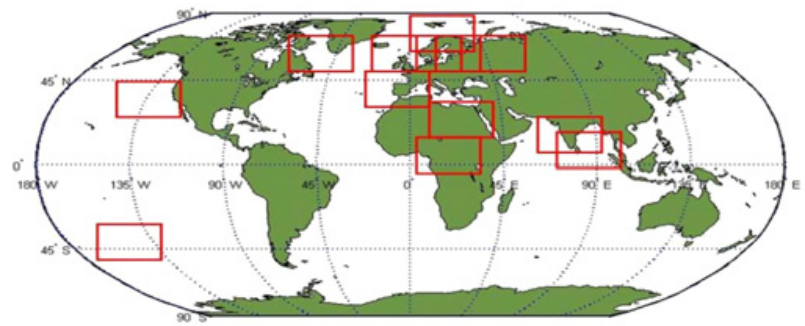

Figure 2. Scheme of the areas selected to calculate the BTDs as a function of the cloud type.

applied to MODIS images the difference between the emissivity retrieved by the procedure proposed and the emissivity provided by MODIS is consistent with the validations, i.e., the differences are $\leq 0.1$, whenever the emissivity is not too low [13]. However if the emissivity is lower than 0.5 the differences increase up to 0.5 for very low emissivities. For this reason it is very important to identify, at least, pixels of low emissivity because the accuracy of the emissivity retrievals can be too poor to retrieve the temperature with the JEM-EUSO required accuracy. This is precisely the aim of this work: to investigate if the BTDs in $\mathrm{BT}_{11}$ and $\mathrm{BT}_{12}$ are appropriate to identify ice thin clouds, which are the main interest for the EAS reconstruction. Nevertheless the problem of low emissivity retrievals is still being studied.

Once the temperatures are retrieved, then the height can be calculated from the cloud temperature by means of temperature vertical profiles (see Fig. 1). The temperature profiles can be obtained from radiosoundings or global atmospheric models. At this stage radiosoundings close to the area covered by the MODIS image have been used to retrieve the height. Results for water thick clouds, where the temperature retrievals are very accurate, are very good. Global atmospheric models could improve the height retrieval accuracy but it will depend on the accuracy of the data provided by the global model.

\section{Classification of water and ice clouds from BTDs between $\mathrm{BT}_{11}$ and $\mathrm{BT}_{12}$}

Although in the first works cloud classification was carried out with bi-spectral sensors [15], nowadays it is generally performed with multispectral sensors $[16,17]$ since it provides more information. Nevertheless, sincethe IR camera requirements have led to a bi-spectral design, it is worth studying the possibilities to classify clouds to help the IR camera data analysis.

In order to determine if a BTD value can be associated to different types of clouds the BTDs of $\mathrm{BT}_{11}$ and $\mathrm{BT}_{12}$ have been calculated for some MODIS images. Since MODIS provides not only the BTs in our bands of interest but many products such as the cloud phase, cloud emissivity, temperature and pressure, the products of this sensor can be used to establish a relationship between the BTDs and the type of cloud. Twelve images embracing different latitudes, longitudes over the Earth and ocean have been selected to have a wide range of scenarios. In Fig. 2 a diagram showing the distribution of areas used in
Table 1. BTDs values for different types of cloud (phase and emissivity).

\begin{tabular}{|c|c|c|c|c|c|}
\hline \multicolumn{2}{|c|}{ BTD } & \multicolumn{4}{c|}{ Emissivity } \\
\hline \multicolumn{2}{|c|}{ BT $_{\mathbf{1 1}} \mathbf{B T}_{\mathbf{1 2}}$} & $\mathbf{( 0 - 0 . 2 5})$ & $\mathbf{( 0 . 2 5 - 0 . 5 )}$ & $\mathbf{( 0 . 5 - 0 . 7 5 )}$ & $\mathbf{( 0 . 7 5 - 1 )}$ \\
\hline \multirow{3}{*}{ Water } & Mean & 0.43 & 0.50 & 0.57 & 0.32 \\
\cline { 2 - 6 } & Variance & 0.35 & 0.43 & 0.48 & 0.19 \\
\hline \multirow{2}{*}{ Ice } & Mean & 1.77 & 2.37 & 2.02 & 0.97 \\
\cline { 2 - 6 } & Variance & 1.61 & 2.37 & 1.65 & 0.82 \\
\hline \multirow{2}{*}{ Mixed } & Mean & 0.35 & 0.84 & 0.96 & 0.61 \\
\cline { 2 - 6 } & Variance & 0.39 & 0.84 & 0.96 & 0.61 \\
\hline
\end{tabular}

the study is provided, where the red squares are roughly located at the latitude and longitude of the MODIS images.

MODIS cloud phase product classifies the cloudy pixels as water, ice, mixed and uncertain. These 12 images provide 466409, 368710, 92802 and 185104 pixels of water, ice, mixed and uncertain pixels respectively. The BTDs of $\mathrm{BT}_{11}$ and $\mathrm{BT}_{12}$ have been calculated for all those pixels. The mean value of BTDs for each class have been divided in different ranges of emissivity to refine the classification in thin and thick clouds of water, ice and mixed. The BTD mean values for each class and subclass are shown in Table 1.

In Table 1 it is clearly seen that for water and mixed clouds the mean BTD increases with the emissivity except for the $0.75-1$ interval where the emissivity is lower than that of the previous emissivity interval. For ice clouds the emissivity increases in the first two intervals but decreases for the intervals of higher emissivity. Other remarkable point is the high variance values, similar in some cases to the BTD value which makes difficult to relate a BTD value with the type of cloud. However from these values a threshold BTD value can be defined to discriminate ice clouds from water and mixed clouds. Effectively, the BTD values for water phase keep below $0.57 \mathrm{~K}$ for all the emissivity intervals. The threshold value formixed phase can be established in $0.96 \mathrm{~K}$. Considering that the BTDs for water pixels are below $0.57 \mathrm{~K}$, another threshold could be defined next to that value to discriminate water pixels although some confusion with mixed pixels could arise. However, all the BTDs of ice pixels remain above $0.97 \mathrm{~K}$ for all the emissivity values. This result leads to fix a BTD value to discriminate ice from water and mixed phase.

The BTDs have been divided in intervals of $0.5 \mathrm{~K}$ and the number of pixels of each interval has been represented in a histogram. In Fig. 3 the distribution of BTDs for water, ice and mixed pixels (top, centre and bottom) is represented to work out if the thresholds mentioned above could be useful to discriminate water and ice clouds.

In order to select water pixels by means of the BTD, a threshold of BTD $=0.5 \mathrm{~K}$ could be used. In this case all the pixels with BTD $<0.5 \mathrm{~K}$ should be classified as water (pixels under the red clasp in top graph of Fig. 3). However, this selection would include not only water pixels but also ice and mixed pixels (pixels under the red clasp in the middle and bottom graphs of Fig. 3). If a threshold of BTD $=1 \mathrm{~K}$ is used to select ice pixels an equivalent problem arises: one can find many pixels of ice with BTD $>1 \mathrm{~K}$ (pixels under the blue clasp in the middle graph of Fig. 3) but the selection would also include some 

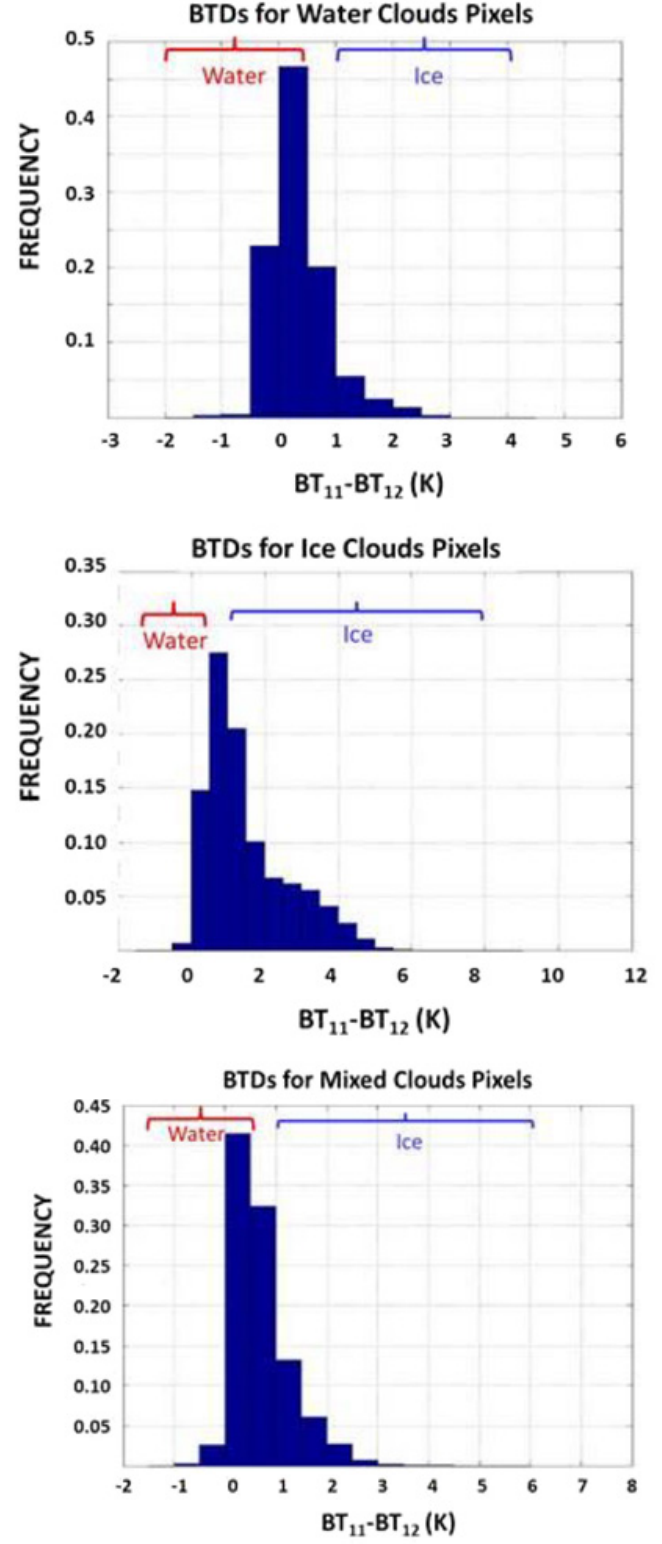

Figure 3. Histograms of BTDs for water, ice and mixed phase pixels (top, centre, bottom). Below the red clasp the pixels classified as water by the threshold below $0.5 \mathrm{~K}$. Below the blue clasp the pixels classified as ice by the threshold above $1 \mathrm{~K}$.

water and mixed pixels (pixels under the blue clasp in the top and bottom graphs of Fig. 3).

Applying these thresholds $(\mathrm{BTD}<0.5 \mathrm{~K}$ - water, $\mathrm{BTD}>1 \mathrm{~K}-$ ice) to the pixels of the 12 MODIS images the classification would result in the percentages shown in Table 2. First row indicates the ratio of pixels of water, ice and mixed classified as water (BTD $<0.5 \mathrm{~K}$ threshold) respect the total number of pixels of its corresponding phase. Second row shows the percentage of pixels of each phase identified as ice when the BTD $>1 \mathrm{~K}$ threshold is used.

The percentages in Table 2 indicate that the first threshold (BTD $<0.5 \mathrm{~K}$ ) would be quite effective in selecting water pixels (70\%). However an important percentage of ice and mixed pixels would be also
Table 2. Percentage of MODIS pixels classified as water or ice by the following thresholds: BTD $<0.5 \mathrm{~K}$ (water), BTD $>1 \mathrm{~K}$ (ice).

\begin{tabular}{|c|c|c|c|}
\hline & \multicolumn{3}{|c|}{ MODIS Clasification } \\
\hline Threshold Classification & Water & Ice & Mixed \\
\hline Water (BTD < $0.5 \mathrm{~K})$ & $70 \%$ & $15 \%$ & $45 \%$ \\
\hline Ice (BTD $>1 \mathrm{~K})$ & $10 \%$ & $55 \%$ & $22 \%$ \\
\hline
\end{tabular}
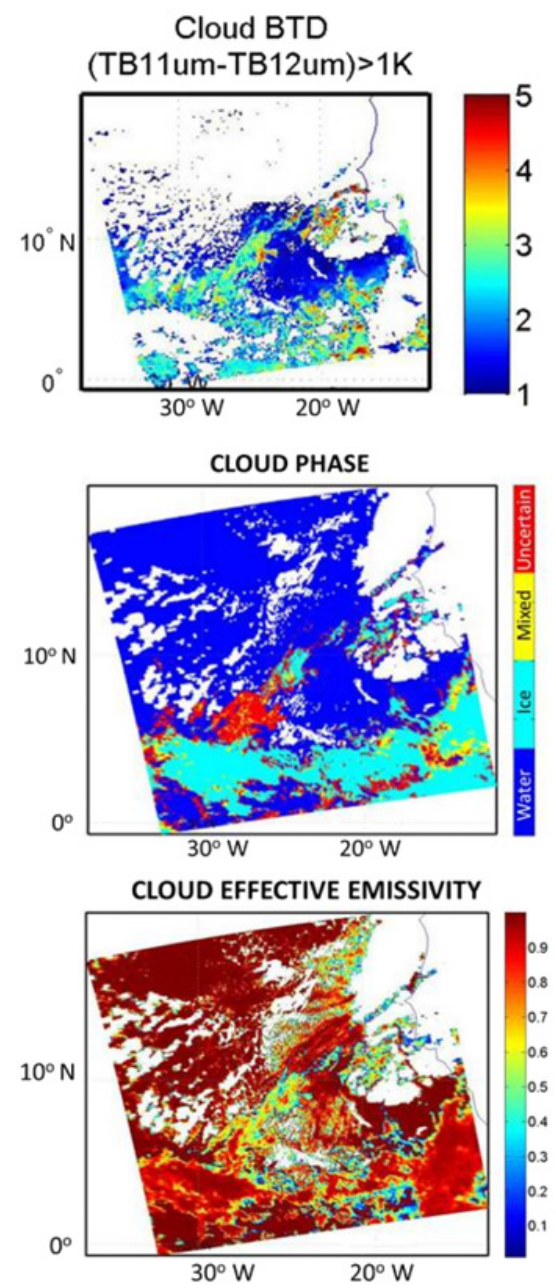

Figure 4. BTDs after applying the BTD $>1 \mathrm{~K}$ threshold. Phase and emissivity are also shown for comparison.

selected as water ( $15 \%$ and $45 \%$ respectively). The second threshold (BTD $>1 \mathrm{~K}$ ) is not so effective in selecting ice pixels $(55 \%)$. Nevertheless the percentage of water and mixed pixels erroneously selected as ice is not so high (10\% and $22 \%$ respectively). Considering these results, the threshold of BTD $<0.5 \mathrm{~K}$ does not seem to be very effective to classify water pixels since it would select ice and mixed pixels at the same time. The threshold BTD > $1 \mathrm{~K}$ presents the same problem.

Figure 4 shows an example of classification in a MODIS image. The area is on the Equator $\left(0-20^{\circ} \mathrm{N}\right)$ near Gambia and Senegal Coast $\left(15-35^{\circ} \mathrm{W}\right)$. At the top the BTD image after applying the threshold is shown. 


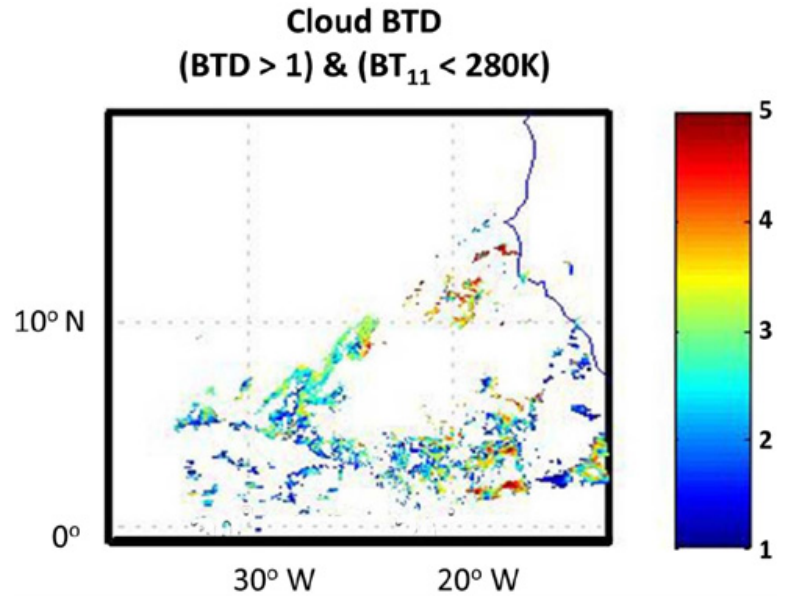

Figure 5. BTDs after applying the $\mathrm{BTD}>1 \mathrm{~K}$ and $\mathrm{BT}_{11}<$ $280 \mathrm{~K}$ thresholds.

Phase and emissivity MODIS products also appear in Fig. 4 to understand which pixels have been selected and discarded. Comparing the three graphs of Fig. 4, it is easy to see that pixels selected by the threshold involve all the phases, although most of the water pixels have been rejected. In addition it can be observed that most of the pixels of high emissivity have been discarded.

The questions that arise now are: what kind of ice pixels have been classified or rejected by the threshold $\mathrm{BTD}>1 \mathrm{~K}$, thin or thick? Is there any other criterion to eliminate water and mixed pixels of the selection? Since the BTDs criteria are not very effective to classify water and ice clouds, could we at least identify thin ice clouds?

\section{Identification of ice thin clouds from BTDs and $\mathrm{BT}_{11}$ thresholds}

Since water clouds are in general lower and warmer clouds, a new selection criterion based on a temperature threshold has been appliedto eliminate water pixels. The thresholdis applied to $\mathrm{BT}_{11}\left(\mathrm{BT}_{11}<280 \mathrm{~K}\right)$. Therefore, after applying the criterion $\mathrm{BTD}>1 \mathrm{~K}$, pixels with $\mathrm{BT}_{11}<280 \mathrm{~K}$ are also rejected. This additional $\mathrm{BT}_{11}$ threshold has been applied to the example studied in section 3. As can be appreciated from Fig. 5 water pixels are definitively discarded and ice pixels with low emissivity are kept.

Table 3 summarizes the effect of applying both criteria to the MODIS image under study. The first column shows the number of pixels classified by MODIS as water or icebefore applying the criteria. The second column displays the number of pixels of each phase that remain after the first criterion application. In the third column the pixels persisting after the second criterion are represented. Data in Table 3 prove that first threshold eliminates water and some ice pixels. It has been checked that the emissivity of rejected ice pixels is high. Second criterion reduces the water pixels drastically but preserves the ice pixels selected by the first criterion. Summarizing: the effect of applying the proposed criteria is to select ice pixels of low emissivity, i.e., ice thin clouds.
Table 3. Number of pixels of water and ice before applying the criteria, after first criterion and after second criterion (first, second and third column respectively).

\begin{tabular}{|l|c|c|c|}
\hline Number of Pixels & \multicolumn{3}{|c|}{ Classification Criteria } \\
\hline Pixel Phase & MODIS & BTD > 1 K & B T $_{\mathbf{1 1}}<\mathbf{2 8 0}$ K \\
\hline Water pixels & 65966 & 34510 & 553 \\
\hline Ice pixels & 18359 & 12537 & 11970 \\
\hline
\end{tabular}

\section{Conclusions}

In this work an analysis of the capabilities of the JEMEUSO IR camera to classify clouds has been carried out. As expected the water and ice classification based only on the BTDs is not effective enough, although depending on the accuracy requirements it could be used. However considering another threshold criterion for the $\mathrm{BT}_{11}$ can improve significantly the results.

In this work the threshold proposed has been addressed to identify thin ice clouds. After applying both thresholds $\mathrm{BTD}>1 \mathrm{~K}$ and $\mathrm{BT} 11<280 \mathrm{~K}$ to a MODIS image it has been demonstrated that this combination is able to identify ice thin clouds. Nevertheless these thresholds could be refined in the future to improve the identification. Moreover other thresholds will be investigated in the future to increase the effectiveness of the water/ice classification.

To conclude: the procedure proposed to retrieve the cloud height is giving very good results. The temperature and height obtained for thick water clouds has been validated and the comparison with MODIS products demonstrates that the accuracy is very good, considering JEM-EUSO requirements. For thin clouds the height and temperature retrieval need the accurate estimation of the emissivity. The LUT-based procedure to estimate the emissivity has been also validated and applied to real scenarios (MODIS images). The comparison with the MODIS emissivity product confirms that the retrieved emissivities are accurate enough whenever the emissivity is higher than 0.5 . Below 0.5 the accuracy of the emissivity retrieval decreases with the emissivity. Although we keep studying this problem, in this work it has been demonstrated that the threshold criteria for BTDs and $\mathrm{BT}_{11}$ are useful to identify at least these problematic situations of low emissivity in order to consider carefully the accuracy of the emissivity retrieval.

Authors would like to acknowledge to the JEM-EUSO members for supporting encouraging us in this work and letting us to be part of the consortium. JEM-EUSO consortium is being funded by: Basic Science Interdisciplinary Research Projects of RIKEN and JSPS KAKENHI Grant (22340063, 23340081, and 24244042), by the Italian Ministry of Foreign Affairs, General Direction for the Cultural Promotion and Cooperation, by the 'Helmholtz Alliance for Astroparticle Physics HAP' funded by the Initiative and Networking Fund of the Helmholtz Association, Germany, and by Slovak Academy of Sciences MVTS JEM-EUSO as well as VEGA grant agency project 2/0081/10. The Spanish Consortium involved in the JEM-EUSO Space Mission is funded by MICINN under projects AYA2009-06037-E/ESP, AYA-ESP 
2010- 19082, AYA2011-29489-C03-01, AYA2011-29489-C0303, AYA2012-39115-C03-01, AYAESP2011- 29489-C03, AYA-ESP2012-39115-C03-02, CSD2009- 00064 (Consolider MULTIDARK) and by Comunidad de Madrid (CAM) under project S2009/ESP-1496.

\section{References}

[1] A. Arking, J. D. Childs, J. Climate Appl. Meteor., 24, 322-333 (1985)

[2] T. Nakajima, M. D. King, J. Atmos. Sci. 47, 1878-1893 (1990)

[3] T.Y. Nakajima, T. Nakajima, J. Atmos. Sci., 52, 4043- 4059 (1995)

[4] R.W. Saunders, K.T. Kriebel, Int. J. Remote Sensing 9, 123-150 (1988)

[5] H.J. Lutz, EUMETSAT Tech. Department Tech. Memo. No. 4, pp 26 (1999)

[6] J.H. Adams et al., Astropart. Phys. 44, 76-90 (2013)

[7] G. Sáez Cano, J.A. Morales de los Ríos, K. Shinozaki, S. Briz, H. Prieto, L. del Peral, J. HCarretero, A.J. de Castro, F. Cortés, F. Lopez, A. Neronov, S. Wada, M.D. Rodríguez Frías, for the JEM-EUSO Collaboration, J. Phys.: Conf. Ser. 375 052010 (2012)

[8] A. Neronov, S. Wada, M.D. Rodríguez-Frías, J.A. Morales de los Ríos, G. SáezCano, H. Prieto, J. Piñeiro, L. del Peral, J. Hernández, N. Pacheco Gómez, M.D. Sabau, T. Beleguer, C. González, M. Reina, S. Briz, A.J. de Castro, F. Cortés, F. López, G. Herrera, J. Licandro, E. Joven, M. Serra, O.Vaduvescu, A. Anzalone, F. Isgrò, R. Cremonini, C. Cassardo, A. Maurissen, C. Urban, T. Ogawa, K. Shinozaki, "Atmospheric Monitoring System of JEM-EUSO, theProceedings of the 32nd International Cosmic Ray Conference, 6, 338-342 (2011)

[9] S. Toscano, L. Valore, A. Neronov, F. Guarino, F. for the JEM-EUSO Collaboration, the Proceedings of the33rd International Cosmic Ray Conference, 1, 143-147 (2013)

[10] J.A. Morales de los Ríos, G. Sáez-Cano, H. Prieto, L. del Peral, J. Pineiro, K. Shinozaki, J. Hernández, N. Pacheco, M.D. Sabau, T. Belenguer, C. Gonzalez Alvarado, M. Reina, S. Briz, A.J.. de Castro, F. Cortés, F. Lopez, J. Licandro, E. Joven, M. Serra, O. Vaduvescu, S. Wada, K. Tsuno, O. Catalano, A. Anzalone, M. Casolino, M.D. Rodríguez-Frías, forthe JEM-EUSO Collaboration. theProceedings of the32nd International CosmicRayConference, Beijing, 1, 147-150 (2011)

[11] A. Anzalone, M. Bertaina, S. Briz, R. Cremonini, F. Isgrò; for the JEM-EUSO Collaboration.the Proceedings of the $33^{\text {rd }}$ International Cosmic Ray Conference, The Astroparticle Physics Conference. Río de Janeiro, 1, 127-131 (2013).

[12] M.D. Rodriguez Frias, J. Licandro, M.D. Sabau, M. Reyes, T. Belenguer, M.C. GonzalezAlvarado, E. Joven, J.A. Morales de los Rios, M. SaezPalomino, H. Prieto-Alfonso, G. Saez Cano, J. H. Carretero, S. Perez Cano, L del Peral forthe JEM-EUSO Collaboration the Proceedings of the 33rd International Cosmic Ray Conference, Rio de Janeiro, 1, 139-142 (2013)

[13] S. Briz, A.J. de Castro, I. Fernández-Gómez, I. Rodríguez, F. López for JEM-EUSO Collaboration these proceedings, Padova (2014)

[14] S. Briz, A.J. de Castro, I. Fernández-Gómez, I. Rodríguez, F. López, J. Appl. Remote Sens. 8, 084990-1-084990-15 (2014)

[15] T.A. Inoue, J. Geophys. Res, 92 (D4), 3991-4000 (1987)

[16] S. Platnick, M.D. King, S.A. Ackerman, W.P. Menzel, A. Baum, C.J. Riédi, R.A. Frey, IEEE Transactions on Geoscience and Remote Sensing, 41, 459-473 (2003)

[17] H. J. Lutz, T. Inoue, J. Schmetz, J. Meteor. Soc. Japan 81, 623-631 (2003) 